\title{
EDITORIAL
}

\section{Nutritional rehabilitation: from pulmonary cachexia to sarcoPD}

\author{
A.M.W.J. Schols
}

E vidence is accumulating that disease burden in chronic obstructive pulmonary disease (COPD) is determined by not only pulmonary impairment but the fact that skeletal muscle pathology and cardiovascular disease also contribute to decreased physical performance and increased mortality. An integrated view of the decline in function of multiple organs as a disturbed homeostatic network resulting from smoking, suboptimal diet, inactivity and disease susceptibility has not yet been conceptualised in COPD management but could lead to a shift in clinical medicine and public health towards personalised lifestyle.

Research into the pathogenesis and treatment of skeletal muscle pathology in pulmonary cachexia to alleviate progressive disability has been the subject of intensive research during the past decade. While the anabolic and metabolic potential of multimodal nutritional rehabilitation strategies, including exercise, protein-calorie supplementation, polyunsaturated fatty acids and/or anabolic agents, has been demonstrated in the short-term [1-3], efficacy in pulmonary cachexia is limited by the absence of long-term strategies to maintain physical fitness in advanced disease and by adverse effects of persistent inflammation on muscle maintenance [4, 5]. Experimental research rapidly advances understanding of the molecular regulation of muscle protein synthesis and breakdown, providing new leads for nutritional and pharmacological modulation. Translation of this research into either generic lifestyle strategies or tailored interventions is currently limited by insufficient insight in the regulation of muscle protein turnover during the course of the disease, ranging from the susceptible smoker to chronic respiratory failure, and from onset to recovery of acute exacerbations. Similarly, it is as yet unclear whether a decreased skeletal muscle oxidative capacity as observed in advanced COPD [6] is merely an adaptive response to long-term sedentary behaviour and smoking, or reflects mitochondrial dysfunction that may interfere with skeletal muscle anabolism and insulin signalling, and thus accelerates muscle wasting and increases susceptibility to cardiovascular disease.

STATEMENT OF INTEREST: A statement of interest for A.M.W.J. Schols can be found at www.erj.ersjournals.com/misc/statements.dtl

CORRESPONDENCE: A.M.W.J. Schols, Dept. of Respiratory Medicine, NUTRIM School for Nutrition, Toxicology and Metabolism, Maastricht University Medicial Centre, P.0. Box 5800, 6202 AZ Maastricht, The Netherlands. Fax: 31 433875046. E-mail: a.schols@pul.unimaas.nl
Atherosclerotic cardiovascular disease is a leading cause of morbidity and mortality in the general population. Based on many ongoing observational investigations, hypercholesterolaemia, hypertension and diabetes mellitus are well-established causes of the cardiovascular epidemic in the general population [7]. These risk factors are accentuated by obesity [8]. Remarkably, in chronic diseases (including COPD) and in advanced age, a reverse epidemiology is observed because in these populations, mortality risk is not increased but is decreased in the overweight and in obesity relative to normal weight patients [9]. The obesity paradox in COPD could be explained by recent body composition studies consistently showing that muscle wasting is a better predictor of mortality in COPD than body mass index and that wasting is not limited to cachexia and end-stage disease but also occurs in a substantial proportion of patients with moderate airflow obstruction [10,11]. The latter group is characterised by a shift in body composition towards "hidden" muscle wasting with relative fat abundance that resembles sarcopenia in advanced age ("sarcoPD") [11]. In chronic heart failure, other surrogates of cardiovascular risk, such as fat abundance, hypercholesterolaemia and hypertension, are paradoxically associated with greater instead of lower survival [9]. In respiratory disease, limited data are available regarding the prognostic value of cardiovascular biomarkers, with the exception of the inverse and graded association between serum cholesterol level and COPD [12].

Large epidemiological studies have reported that in addition to a high prevalence of traditional risk factors in COPD, including smoking, sedentary behaviour and low socio-economic class, airflow limitation is an independent risk factor for cardiovascular disease [13]. It is tempting to speculate that the observed association between forced expiratory volume in one second and cardiovascular mortality not only represents the influence of respiratory impairment but also of "accelerated ageing", as reflected by progressive muscle wasting that is masked by preservation of fat mass. This hypothesis is supported by a remarkable similarity in denominators of enhanced cardiovascular disease risk, muscle wasting and adaptive responses to physical inactivity, including systemic inflammation, oxidative stress and hypoxia, as nicely reviewed recently by MACNEE et al. [13]. Susceptibility of the sarcopenic COPD phenotype is not only increased by adverse effects of muscle wasting on physical functioning and mortality, but fat abundance may also enhance cardiometabolic risk. In a previous issue of the European Respiratory Journal, FRANSSEN et al. [14] report that in contrast to the metabolic syndrome, 
sarcopenia in COPD is not accompanied by an abnormal whole body lipolytic rate but that homeostatic control of lipolysis is altered as reflected by hyperinsulinaemia. Based on measurement of fasting glucose, insulin and homeostatic modelling techniques, BOLTON et al. [15] reported greater insulin resistance in normal weight patients with COPD compared to healthy control subjects, which was related to systemic inflammation.

To improve vitality and compress morbidity of COPD the current literature already provides a strong rationale to broaden the scope of nutritional rehabilitation from pulmonary cachexia to "sarcoPD". Evaluation of cardiovascular biomarker modifiability after different nutritional rehabilitation strategies in this COPD phenotype could be a unique clinical intervention model to elucidate the apparent risk factor paradox in chronic wasting disease.

The conclusion of this risk-benefit balance? There is no time to waste muscle in chronic obstructive pulmonary disease.

\section{REFERENCES}

1 Schols AM, Soeters PB, Mostert R, Pluymers RJ, Wouters EF. Physiologic effects of nutritional support and anabolic steroids in patients with chronic obstructive pulmonary disease. A placebo-controlled randomized trial. Am J Respir Crit Care Med 1995; 152: 1268-1274.

2 Broekhuizen R, Wouters EFM, Creutzberg EC, WelingScheepers CAPM, Schols AMWJ. Polyunsaturated fatty acids improve exercise capacity in chronic obstructive pulmonary disease. Thorax 2005; 60: 376-382.

3 Creutzberg EC, Wouters EFM, Mostert R, WelingScheepers CAPM, Schols AMWJ. Efficacy of nutritional supplementation therapy in depleted patients with chronic obstructive pulmonary disease. Nutrition 2003; 19: 120-127.

4 Bolton CE, Broekhuizen R, Ionescu AA, et al. Cellular protein breakdown and systemic inflammation are unaffected by pulmonary rehabilitation in COPD. Thorax 2007; 62: 109-114.
5 Creutzberg EC, Schols AMWJ, Weling-Scheepers CAPM, Buurman WA, Wouters EFM. Characterization of nonresponse to high caloric oral nutritional therapy in depleted patients with chronic obstructive pulmonary disease. Am J Respir Crit Care Med 2000; 161: 745-752.

6 Gosker HR, Zeegers M, Wouters FM, Schols AMWJ. Muscle fibre type shifting in the vastus lateralis of patients with COPD is associated with disease severity: a systematic review and meta-analysis. Thorax 2007; 62: 944-949.

7 Grundy S. Age as a risk factor: you are as old as your arteries. Am J Cardiol 1999; 83: 1455-1457.

8 Pardo Silva MC, De Laet C, Nusselder WJ, et al. Adult obesity and number of years lived with and without cardiovascular disease. Obesity 2006; 14: 1264-1273.

9 Kalantar-Zadeh K, Horwich TB, Oreopoulos A, et al. Risk paradox in wasting diseases. Curr Opin Clin Nutr Metab Care 2007; 10: 433-442.

10 Vestbo J, Prescott E, Almdal T, et al. Body mass, fat-free body mass, and prognosis in patients with chronic obstructive pulmonary disease from a random population sample: findings from the Copenhagen City Heart Study. Am J Respir Crit Care Med 2006; 173: 79-83.

11 Schols AM, Broekhuizen R, Weling-Scheepers CA, Wouters EF. Body composition and mortality in chronic obstructive pulmonary disease. Am J Clin Nutr 2005; 82: 53-59.

12 Iribarren C, Jacobs DR Jr, Sidney S, et al. Serum total cholesterol and risk of hospitalization, and death from respiratory disease. Int J Epidemiol 1997; 26: 1191-1202.

13 MacNee W, Maclay J, McAllister D. Cardiovascular injury and repair in chronic obstructive pulmonary disease. Proc Am Thoracic Soc 2008; 5: 824-833.

14 Franssen FM, Sauerwein HP, Rutten EP, Wouters EF, Schols AM. Whole body resting and exercise-induced lipolysis in sarcopaenic patients with COPD. Eur Respir J 2008; 32: 1466-1471.

15 Bolton CE, Evans M, Ionescu AA, et al. Insulin resistance and inflammation - a further systemic complication of COPD. COPD 2007; 4: 121-126. 